\title{
Monotone Principle of Forked Points and Its Consequences
}

\author{
Milan R. TASKOVIĆ
}

\begin{abstract}
This paper presents applications of the Axiom of Infinite Choice: Given any set $P$, there exist at least countable choice functions or there exist at least finite choice functions. The author continues herein with the further study of two papers of the Axiom of Choice in order by E. Zermelo [Neuer Beweis für die Möglichkeit einer Wohlordung, Math. Annalen, 65 (1908), 107-128; translated in van Heijenoort 1967, 183-198], and by M. Tasković [The axiom of choice, fixed point theorems, and inductive ordered sets, Proc. Amer. Math. Soc., 116 (1992), 897-904]. Monotone principle of forked points is a direct consequence of the Axiom of Infinite Choice, i.e., of the Lemma of Infinite Maximality! Brouwer and Schauder theorems are two direct censequences of the monotone principle od forked points.
\end{abstract}

\section{INTRODUCTION AND HISTORY}

Let $X:=(X, M)$ be a topological space and $T: X \rightarrow X$, where $M$ : $X \rightarrow \mathbb{R}_{+}^{0}:=[0,+\infty)$. In connection with this, in 1985 we investigated the concept of TCS-convergence in a space $X$, i.e., a topological space $X$ satisfies the condition of local TCS-convergence iff $x \in X$ and if $M\left(T^{n} x\right) \rightarrow 0$ $(n \rightarrow \infty)$ implies that $\left\{T^{n}(x)\right\}_{n \in \mathbb{N}}$ has a convergent subsequence.

Theorem 1.1. (Localization Monotone Principle, Tasković [1990, Th. 1]). Let $T$ be a mapping of a topological space $X:=(X, M)$ into itself, where $X$ satisfies the condition of local TCS-convergence. Suppose that there exists a mapping $\varphi: \mathbb{R}_{+}^{0} \rightarrow \mathbb{R}_{+}^{0}$ such that

( $\varphi) \quad\left(\forall t \in \mathbb{R}_{+}:=(0,+\infty)\right)\left(\varphi(t)<t \quad\right.$ and $\left.\quad \limsup _{z \rightarrow t+0} \varphi(z)<t\right)$

2000 Mathematics Subject Classification. Primary 01A55, 01A60, 03-03, 03E25, 0403, 04A25; Secondary: 47H10, 05A15, 54H25.

Key words and phrases. The Axiom of Infinite Choice, Zermelo's Axiom of Choice, Lemma of Infinite Maximality, Zorn's lemma, Foundation of the Fixed Point Theory, Fixed point theorems, Topological spaces, Forked monotone principles, Brouwer theorem, Schauder theorem, Forks Theory. 
and the following inequality holds in the form as

$$
M(T(x)) \leq \varphi(M(x)) \quad \text { for every } x \in X,
$$

where $M: X \rightarrow \mathbb{R}_{+}^{0}$ is a T-orbitally lower semicontinuous function and $M(u)=0$ implies $T(u)=u$. Then $T$ has at least one fixed point in $X$.

For $x \in X$ the set $\sigma(x, \infty):=\left\{x, T x, T^{2} x, \ldots\right\}$ is called the orbit of $x$. A function $f$ mapping $X$ into the reals is $f$-orbitally lower semicontinuous at $p$ if $\left\{x_{n}\right\}_{n \in \mathbb{N}}$ is a sequence in $\sigma(x, \infty)$ and $x_{n} \rightarrow p(n \rightarrow \infty)$ implies that $f(p) \leq \lim$. inf $f\left(x_{n}\right)$. A space $X$ is said to be $T$-orbitally complete iff every Cauchy sequence which is contained in $\sigma(x, \infty)$ for some $x \in X$ converges in $X$.

Let $X:=(X, A)$ be a topological space and $T: X \rightarrow X$, where $A$ : $X \times X \rightarrow \mathbb{R}_{+}^{0}$. In 1985 year we investigated the concept of TCS-convergence in a space $X$, i.e., a topological space $X$ satisfies the condition of TCSconvergence iff $x \in X$ and if $A\left(T^{n} x, T^{n+1} x\right) \rightarrow 0(n \rightarrow \infty)$ implies that $\left\{T^{n}(x)\right\}_{n \in \mathbb{N}}$ has a convergent subsequence. As an immediate consequence of Theorem 1.1 we have the following statement on topological spaces.

Theorem 1.2. (Monotone Principle, Tasković [1990, Th. 2]). Let T be a mapping of a topological space $X:=(X, A)$ into itself, where $X$ satisfies the condition of TCS-convergence. Suppose that there exists a mapping $\varphi$ : $\mathbb{R}_{+}^{0} \rightarrow \mathbb{R}_{+}^{0}$ such that $(\varphi)$ and

$$
A(T x, T y) \leq \varphi(A(x, y)) \quad \text { for all } x, y \in X,
$$

where $x \mapsto A(x, T(x))$ is a T-orbitally lower semicontinuous function and $A(u, v)=0$ implies $u=v$. Then $T$ has a unique fixed point $\zeta \in X$ and $T^{n}(x) \rightarrow \zeta$ as $n \rightarrow \infty$ for each $x \in X$.

Proof. Let $M(x):=A(x, T(x))$, then it is easy to see that $A$ and $\varphi$ satisfy all the required hypotheses in Theorem 1.1. Uniqueness follows immediately from condition (2). The proof is complete.

Survey of facts. For the preceding monotone principles, specially for Localization Monotone Principle of Fixed Point, James Dugundji, in the letter for me of October 5 in 1984 year, briefly among the rest writes that he is convinced of the role of Localization Monotone Principle in the fixed point theory (and nonlinear functional analysis).

This opinion of J. Dugundji has been confirmed many a time, via various phenomena, as one can see from many results proven in nonlinear analysis and nature.

In this paper we considered and formulated some new monotone principles for fixed points and for fixed apices as a new way in the nonlinear functional analysis.

We notice that Djuro Kurepa in 1971, first version of my Monotone Principle of Fixed Point, has been sent to Professor J ean Leray (Paris) for the opinion. Some of Leray's ideas I am to realize in several published papers. In general form for the first time, fundamental elements of Monotone Principle I give in: Proc. 
Amer. Math. Soc., 94 (1985, Theorem 2), 427-432. For later facts on this see: Tasković [1990].

History of TCS-convergence. For the first time in 1985 I introduced the conditions of TCS-convergence and local TCS-convergence with the intention to transmit it to the properties of Cauchy sequence from metric spaces on topological spaces, see: Tasković [1990].

This conceptions are very operational and useful for "calculation" on topological spaces. In this sense after this viewpoint appears in most of my papers and books from fixed point theory (see: Tasković [1986], [1990] and [2001]). We can briefly say, in connection with this, that the results of forked points are based on RBSconvergence and BCS-convergence. It is a new viewpoint which is an extension of the TCS-convergence.

At the interval of the next seven years more authors have considered appearance of TCS-convergence as a special case od the property TCS-convergence, precisely, in this way, $d$-completeness of topological spaces, see: Hicks [1992], HicksRhoades [1992], Saliga [1996], and Popa [2002].

A topological space $X$ is an $d$-complete space iff for the function $d: X \times X \rightarrow$ $\mathbb{R}_{+}^{0}$ with the property $d(x, y)=0$ if and only if $x=y$ the following condition

$$
\sum_{n=1}^{\infty} d\left(x_{n}, x_{n+1}\right)<+\infty
$$

implies that the sequence $\left\{x_{n}\right\}_{n \in \mathbb{N}}$ converges in $X$. It is simple to see that the $d$-completeness is only one very special case of the condition of TCS-convergence.

Recently, 10 years next appeared Monotone Principle in 1985, in connection with this Jachymski, Matkowski, and Swiątkowski [Journal of Applied Analysis, 1 (1995), 125-134, Theorem 1, p. 130] proved a very special case of Monotone Principle of Fixed Point on Hausdorff spaces. For the same also see: Aamri-Moutawakil [2003].

\section{Monotony and Forked Points}

Further, by the "Axiom of Infinte Choice" we mean a statement in the following form as: Given any set $S$, there exist at least countable choice functions or there exist at least finite choice functions.

In general, equivalents of the Axiom of Infinite Choice appear frequently in almost all branches of mathematics in a large variety of different forms.

In this part of the paper we present an equivalent form of the Axiom of Infinite Choice which is expressible in the following sense.

Lemma 2.1. (Lemma of Infinite Maximality, Tasković [2012]). Let P be an inductive partially ordered set with ordering $\preccurlyeq$, then $P$ has at least countable maximal elements or $P$ has at least finite maximal elements.

In this part on topological spaces we formulate new monotone principles of forked points. This text continues the further study of the papers by M. R. Tasković [A monotone principle of fixed points, Proc. Amer. Math. Soc., 94 (1985), 427-432, Lemma 2 and Theorem 2], and M. R. Tasković [Some new principles in fixed point theory, Math. Japonica,35 (1990), 645-666]. New monotone principles to include some recent results of author, which contains, as special cases, some results of S. Banach, J. Dugundji, A. Granas, F. Browder, D. W. Boyd, J. S. Wong, J. 
Caristi, T. L. Hicks, B. E. Rhoades, B. Fisher, S. Massa, Dj. Kurepa, M. Kwapisz, W. Kirk, S. Park, M. Krasnoselskij, V. J. Stečenko, T. Kiventidis, I. Rus, K. Iséki, J. Walter, J. Daně̌, A. Meir, E. Keeler, S. Romaguera, J. Matkowski, K. Goebel, L. Collatz, J. Istrăţescu, A. Miczko, B. Palczewski, C. S. Wong, and many others.

Further, in this part we introduced a fundamental result of a new forks theory which unified and connected three known theories on fixed point, transversality and von Neumann's minimax theory. In classical von Neumann's theory fundamental notions is saddle point. In new general convex forks theory its role plays transversal and forked points. In this sense we formulate a new way in nonlinear functional analysis.

Let $X$ be an arbitrary nonempty set, $T$ be a mapping from $X$ into $X$, and $P:=(P, \preccurlyeq)$ a nonempty partially ordered set. A mapping $f: X \rightarrow P$ (or $f: X \rightarrow X$ ) has a forked point (or furcate point) $p \in X$ if the following equality holds in the form

$$
f(p)=f(T p) \text { for some } p \in X \text {; }
$$

frequently, we say that in this case (Ra), the mapping $f: X \rightarrow P$ or $f: X \rightarrow X$ has a pair $(p, T p)$ of bifurcation points, or that $T: X \rightarrow X$ has a forked (or forks) point $p \in X$.

We notice that many problems in nonlinear functional analysis (as and in the fixed point theory) are reducible to the existence of forked points of certain mappings.

Further, let $P:=(P, \preccurlyeq)$ be a partially ordered set with a minimum (or with the property that every nonempty subset in $P$ has an infimum) such that every decreasing sequence $\left\{x_{n}\right\}_{n \in \mathbb{N}}$ in $P$ has a limit in $P$, denoted by $\lim _{n \rightarrow \infty} x_{n}$.

In connection with this, we shall introduce the concept of lower ordered RBS-convergence in a topological space $X$ for $B: X \rightarrow P$, i.e., a topological space $X$ satisfies the condition of lower ordered RBS-convergence iff $\left\{a_{n}(x)\right\}_{n \in \mathbb{N}}$ is an arbitrary sequence in $X$ with arbitrary $x \in X$ and if $B\left(a_{n}(x)\right) \rightarrow b=b(x) \in P(n \rightarrow \infty)$ implies that $\left\{a_{n}(x)\right\}_{n \in \mathbb{N}}$ has a convergent subsequence $\left\{a_{n(k)}(x)\right\}_{k \in \mathbb{N}}$ which converges to $\xi \in X$, where

$$
B(\xi) \preccurlyeq \inf _{x \in X} \lim _{k \rightarrow \infty} B\left(a_{n(k)}(x)\right) .
$$

In this part of the paper, we apply the technick of maximal elements to the equations of the forks theory. As an immediate consequence of Lemma 2.1 we obtain the following ordered principle.

In this sense, let $X$ be a topological space, Define a relation $\preccurlyeq B$, con on $X$ by the following conditions:

$$
\left\{\begin{array}{lll}
x \preccurlyeq B, \text { con } y & \text { if and only if } \quad B(x) \preccurlyeq B(y), \\
x=_{B, \text { con }} y & \text { if and only if } & B(x)=B(y),
\end{array}\right.
$$

where $B: X \rightarrow P$ is a function with the given conditions.

It is verify that $\preccurlyeq B$,con is a partial ordering (asymmetric and transitive relation) in $X$. The poset $X$ together with this partial ordering, is denoted by $X_{B, c o n}$. 
Theorem 2.1. (Ordering Principle). Let $X$ be a topological space with the poset $X_{B, c o n}$. If $X$ satisfies the condition of lower ordered $R B S$-convergence, then $X_{B, c o n}$ has at least countable or finite minimal elements $z_{k} \in X_{B \text {,con }}$ with $z_{k} \preccurlyeq B$,con $x$ for given $x \in X_{B, \text { con }}$.

Proof. Let $C$ be a chain in $X_{B, c o n}$ and now let $t \in C$ be given. Denote by $\alpha:=\inf \{B(x): x \in C\}$. If $B(m)=\alpha$ for some $m \in C$, then $m$ is a lower bound in C. For, if $x \preccurlyeq B$,con $m$ for some $x \in C \backslash\{m\}$, then $B(x) \preccurlyeq B(m)$, which yields $B(x) \prec \alpha$, which is a contradiction. Therefore, one san assume $B(x) \neq \alpha$ for all $x \in C$. Then the set $M(x, n)$ of all $y \in C$ with $y \preccurlyeq B$,con $x$ and $\alpha \prec B(y) \prec \alpha_{n}\left(\alpha_{n} \rightarrow \alpha\right)$ is nonempty for each $n \in \mathbb{N}$ and $x \in C$. In fact, there is a $y \in C$ satisfying $\alpha \prec \beta(y) \prec \alpha_{n}$, and so $y$ belongs

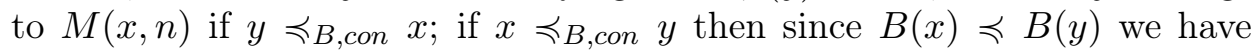
$\alpha \prec B(x) \preccurlyeq B(y) \prec \alpha_{n}$, which shows that $x$ belongs to $M(x, n)$. Let $I$ be a choice function for the family of all nonempty subsets of $C$. Then, by the recursion theorem, there is a sequence $\left\{x_{n}\right\}_{n \in \mathbb{N}}$ in $C$ such that $x_{0}=t$ and $x_{n+1}=I\left(M\left(x_{n}, n\right)\right)$ for $n \in \mathbb{N}$. Since $x_{n+1} \preccurlyeq B$,con $x_{n}$ for all $n \in \mathbb{N}$, we have $B\left(x_{n+1}\right) \preccurlyeq B\left(x_{n}\right)$ for all $n \in \mathbb{N}$, i.e., $\left\{B\left(x_{n}\right)\right\}_{n \in \mathbb{N}}$ converges.

This implies (from lower ordered RBS-convergence) that there exists $\xi \in$ $X_{B, \text { con }}$ such that $B(\xi) \preccurlyeq \cdots \preccurlyeq B\left(x_{n}\right)$ for $n \in \mathbb{N}$. Now let $x \in C$. Then we can find an $i \in \mathbb{N}$ such that $B(\xi) \preccurlyeq B\left(x_{i}\right) \prec \alpha_{i} \preccurlyeq B(x)$. Since $x$ and $x_{i}$ are in the chain $C$, we obtain $\xi \preccurlyeq B$,con $x$. This shows that $\xi$ is a minorant of $C$. By the nature of $C$ (by Lemma of Infinite Maximality) it follows that there is at least countable or finite $z_{k} \in X_{B, c o n}$ which are minimal in $X_{B, c o n}$.

We notice that the proof of this statement is totally an analogy with the former proofs of ordered principles.

As an immediate consequence of Theorem 2.1 (Ordering Principle) we obtain the following result in the forks theory.

Theorem 2.2. (Monotone principle of forked points). Let $T$ be a mapping of a topological space $X$ into itself, where $X$ satisfies the condition of lower ordered RBS-convergence. If

$$
B(T x) \preccurlyeq B(x) \quad \text { for every } \quad x \in X,
$$

then for $T$ there exist at least countable or finite forked points $\xi_{k} \in X$, i.e., then the following equalities hold in the form

$$
B\left(T \xi_{k}\right)=B\left(\xi_{k}\right)=\alpha_{k}:=\inf _{x \in X} \lim _{n \rightarrow \infty} B\left(b_{n}^{k}(x)\right)
$$

for some sequences $\left\{b_{n}^{k}(x)\right\}_{n \in \mathbb{N}}$ in $X$ which converges to the forked points $\xi_{k} \in X$.

Proof. Consider the partially ordered set $X_{B, \text { con }}$ and let $\xi_{k}$ be minimal elements. Using (B), it also following from (fk) that $T x \preccurlyeq B$,con $x$ for all $x \in X$ and $T \xi_{k} \preccurlyeq_{B \text {,con }} \xi_{k}$ in $X_{B, \text { con }}$ and, because $\xi_{k}$ are minimal it follows that $T \xi_{k}={ }_{B, c o n} \xi_{k}$. The proof is complete. 
A brief proof of a special variant of this statement based on some elementary facts may be found in Tasković [2005]. For this, also see Tasković [2001].

Proof of a special case of Theorem 2.2. Let $x$ be an arbitrary point in $X$. Then from the inequality (B) we obtain the following inequalities in the form

$$
\cdots \preccurlyeq B\left(T^{n+1} x\right) \preccurlyeq B\left(T^{n} x\right) \preccurlyeq \cdots \preccurlyeq B(T x) \preccurlyeq B(x)
$$

for every $n \in \mathbb{N} \cup\{0\}$ and for every $x \in X$. Thus, for the sequence $\left\{B\left(T^{n} x\right)\right\}_{n \in \mathbb{N} \cup\{0\}}$ from (3), we obtain that $B\left(T^{n} x\right) \rightarrow b \in P(n \rightarrow \infty)$ with arbitrary $x \in X$. This implies (from the lower ordered RBS-convergence) that its sequence $\left\{T^{n} x\right\}_{n \in \mathbb{N} \cup\{0\}}$ contains a convergent subsequence $\left\{T^{n(k)}(x)\right\}_{k \in \mathbb{N}}$ with a limit point $\xi \in X$. Since $X$ satisfies the condition of lower ordered RBS-convergence, from (3), we have

$$
\begin{gathered}
\alpha:=\inf _{x \in X} \lim _{n \rightarrow \infty} B\left(T^{n} x\right) \preccurlyeq \lim _{n \rightarrow \infty} B\left(T^{n} \xi\right) \preccurlyeq \cdots \\
\cdots \preccurlyeq B(T \xi) \preccurlyeq B(\xi) \preccurlyeq \inf _{x \in X} \lim _{k \rightarrow \infty} B\left(T^{n(k)} x\right)=\alpha ;
\end{gathered}
$$

i.e., $B(T \xi)=B(\xi)=\alpha$. This means that (Ri) holds, i.e., that the mapping $T: X \rightarrow X$ has a forked point $\xi \in X$, where the existing sequence $\left\{b_{n}(x)\right\}_{n \in \mathbb{N}}$, de facto, is the preceding subsequence of the sequence of iterates $\left\{T^{n(k)} x\right\}_{k \in \mathbb{N}}$. The proof is complete.

Let $X$ be an arbitrary nonempty set, $T: X \rightarrow X$, and $P:=(P, \preccurlyeq)$ be a nonempty poset. A mapping $f: X \rightarrow P$ (or $T: X \rightarrow X$ ) has a $k$-forked point (or $k$-furcate point) $p \in X$ if for arbitrary fixed integer $k \geqslant 1$ the following equalities hold in the form

$$
f\left(T^{k} p\right)=\cdots=f(T p)=f(p) \text { for some } p \in X ;
$$

Frequently, we say that in this case (Rk), the mapping $f: X \rightarrow P$ or $f: X \rightarrow X$ has cycle or $k$-pair $\left(p, T p, \ldots, T^{k} p\right)$ of bifurcation points, or that $T: X \rightarrow X$ has a $k$-forked or a $k$-forks point $p \in X$.

In connection with this, from the proof of Theorem 2.2, we obtain, as a direct extension of the preceding result, the following general statement.

Theorem 2.3. (Existence of $k$-forked points). Let $T$ be a mapping of a topological space $X$ into itself, where $X$ satisfies the condition of lower ordered $R B S$-convergence. If

$$
B(T x) \preccurlyeq B(x) \quad \text { for every } \quad x \in X,
$$

then for $T$ there exist at least countable or finite $k$-forked points $\xi_{t} \in X$, i.e., then the following equalities hold in the form

$$
B\left(T^{k} \xi_{t}\right)=\cdots=B\left(T \xi_{t}\right)=B\left(\xi_{t}\right)=\alpha_{t}:=\inf _{x \in X} \lim _{n \rightarrow \infty} B\left(b_{n}^{t}(x)\right)
$$

for an arbitrary fixed integer $k \geqslant 1$ and for some sequence $\left\{b_{n}^{t}(x)\right\}_{n \in \mathbb{N}}$ in $X$ which converges to $\xi_{t} \in X$. 
Interpretation and facts. We notice, first, that Theorem 2.1 hold even we are to make weaker the condition of lower ordered RBS-convergence, in the sense that this condition holds only for iteration sequences.

In this sense, let $X$ be an arbitrary nonempty set, $T: X \rightarrow X, P:=(P, \preccurlyeq)$ be a nonempty poset, and $B: X \rightarrow P$. A topological space $X$ satisfies the condition of orbital lower ordered RBS-convergence iff $\left\{T^{n}(x)\right\}_{n \in \mathbb{N}}$ is an arbitrary iteration sequence in $X$ with arbitrary $x \in X$ and if $B\left(T^{n}(x)\right) \rightarrow b=b(x) \in P(n \rightarrow \infty)$ implies that $\left\{T^{n}(x)\right\}_{n \in \mathbb{N}}$ has a convergent subsequence $\left\{T^{n(k)}(x)\right\}_{k \in \mathbb{N}}$ which converges to $\xi \in X$, where

$$
B(\xi) \preccurlyeq \inf _{x \in X} \lim _{k \rightarrow \infty} B\left(T^{n(k)}(x)\right) .
$$

Also, we shall introduce the concept of RBS-completeness in a space $X$ for a function $B: X \rightarrow$ $P$, i.e., a topological space $X$ is called lower ordered RBS-complete (orbital lower ordered $R B S$-complete) iff $\left\{a_{n}(x)\right\}_{n \in \mathbb{N}}$ is an arbitrary sequence (an arbitrary iteration sequence) in $X$ with arbitrary $x \in X$ and if $B\left(a_{n}(x)\right) \rightarrow b=b(x) \in P$ as $n \rightarrow \infty$ implies that $\left\{a_{n}(x)\right\}_{n \in \mathbb{N}}$ has a convergent subsequence in $X$.

On the other hand, a function $B: X \rightarrow P$ is lower ordered RBS-continuous (orbital lower ordered $R B S$-continuous) at $p \in X$ iff $\left\{a_{n}(x)\right\}_{n \in \mathbb{N}}$ is an arbitrary sequence (an arbitrary iteration sequence) in $X$ with arbitrary $x \in X$ and if $a_{n}(x) \rightarrow p(n \rightarrow \infty)$ implies that

$$
B(p) \preccurlyeq \inf _{x \in X} \lim _{n \rightarrow \infty} B\left(a_{n}(x)\right) .
$$

Second, we are now in a position to formulate the following explanations of the preceding theorems as corresponding equivalent forms:

Theorem 2.4. Let $T$ be a mapping of a topological space $X$ into itself and let $X$ be orbital lower ordered RBS-complete. If $(B)$ holds and if $B: X \rightarrow P$ is an orbital lower ordered RBS-continuous map, then for $T$ there exist at least countable or finite $k$-forked points $\xi_{t} \in X$.

This result is contained in Theorem 2.2 as the case for $k=1$, i.e., for the case of a forked point. In this sense we obtain an immediate result for $P:=\mathbb{R}_{+}^{0}$ and $\preccurlyeq:=\leq$.

In this sense, we shall introduce the concept of lower BCS-convergence in a topological space $X$ for $B: X \rightarrow \mathbb{R}_{+}^{0}$, i.e., a topological space $X$ satisfies the condition of lower BCS-convergence (orbital lower BCS-convergence) if $\left\{a_{n}(x)\right\}_{n \in \mathbb{N}}$ is an arbitrary sequence (an arbitrary iteration sequence) in $X$ with arbitrary $x \in X$ and if $B\left(a_{n}(x)\right) \rightarrow b=b(x) \geqslant 0(n \rightarrow \infty)$ implies that $\left\{a_{n}(x)\right\}_{n \in \mathbb{N}}$ has a convergent subsequence $\left\{a_{n(k)}(x)\right\}_{k \in \mathbb{N}}$ which converges to $\xi \in X$, where

$$
B(\xi) \leqslant \inf _{x \in X} \liminf _{k \rightarrow \infty} B\left(a_{n(k)}(x)\right) .
$$

In connection with this, the preceding result is an extension of our former Localization Monotone Principle of fixed point proved for the first time in Tasković [1985].

A brief proof of a special case of this statement based on some elementary facts may be found in Tasković [2001] and [2002]. For this, also see Tasković [2005].

Annotations. From (B) and Ordering Principle it directly follows that the mapping $T$ of a topological space $X$ into itself on the set $X_{B \text {,con }}$ has at least countable or finite fixed points $\xi_{t} \in X$ as minimal elements of this set.

Even more, from Theorem 2.2 it follows that $T$, as a mapping of a topological space $X$ into itself where $X$ with the property of lower BCS-convergence, has a fixed point $\xi \in X$ if and only if $\xi$ is a minimal element of the set $X_{B \text {,con }}$ for which we have $\xi={ }_{B \text {,con }} T \xi$.

Annotations. A fine illustration for Theorem 2.2 is a well known statement in 1936 which was given by Freudenthal and Hurewicz in the following form: If $(X, \rho)$ is a compact 
metric space and if $T$ is a mapping of $X$ into itself such that

$$
\rho[T(x), T(y)]<\rho[x, y] \quad \text { for all } \quad x, y \in X \quad(x \neq y),
$$

then the mapping $T$ has a unique fixed point $\xi \in X$.

Indeed, first, since $X$ is a compact space it follows that the condition of lower (orbital) BCSconvergence holds. Second, let $B(x):=\rho(x, T x)$, thus applying Theorem 2.2 we have that there exists $\xi \in X$ such that (Ri). But, from (4) for $\xi \neq T \xi$ we obtain

$$
B(\xi)=B(T \xi)=\rho\left[T(\xi), T^{2}(\xi)\right]<\rho[\xi, T(\xi)]=B(\xi),
$$

i.e., we obtain a contradiction. This means that $\xi=T \xi$ for some $\xi \in X$. The uniqueness follows immediately from (4). The proof is complete.

In connection with this statement of Freudenthal-Hurewicz [1936] there exist more extensions. An extension of this statement to give Edelstein [1962] to change the compactness with the following weak condition in the form: if $\left\{T^{n}(x)\right\}_{n \in \mathbb{N}}$ is an arbitrary iteration sequence in $X$ with arbitrary $x \in X$, then he has at least one convergent subsequence in $X$.

On the other hand we notice that this result of Freudenthal and Hurewicz in 1936 appeared, also, at the same year independently by Niemytzki [1936].

These facts are direct examples for the preceding Theorem 2.2. Also, this facts can be great for further considerations specially in the fixed point theory.

Extensions theorems of Brouwer and Schauder. This part is primarily devoted to illustraing the preceding results, thus in the forks theory we obtain extensions of Brouwer and Schauder theorems.

Proposition 2.1. Let $X$ be a convex subset in linear topological space $Y$ and let $T$ be a mapping of $X$ into itself. Then there exists a lower semicontinuous function $B: X \rightarrow \mathbb{R}_{+}^{0}$ such that inequality (B) holds.

Proof. Let $B: X \rightarrow \mathbb{R}_{+}^{0}$ be a convex function with the property $B((x+$ $y) / 2) \geq B(x)$ for all $x, y \in X$, where $B$ is a lower semicontinuous function on the convex set $X$. Then, immediate (B) holds, because $0 \leq B((x+$ $y) / 2)-B(x) \leq B(y)-B(x)$ for all $x, y \in X$. Thus, $B(T(x)) \leq B(x)$ for every $x \in X$, i.e., inequality (B) holds. The proof is complete.

Further, in this section we apply the monotone principle of forked points (for $P:=\mathbb{R}_{+}^{0}$ and $\preccurlyeq:=\leq$ ) to the Schauder's 54th problem in Scottish Book. In this sense we have the following extension and a new solution of Schauder's problme on linear topological spaces. ${ }^{1}$

\footnotetext{
${ }^{1}$ History of Schauder's problem. The most famous of many open problems in nonlinear analysis is Schauder's problem (in Scottish book, problem 54). For some answers on this problem see papers of: Tychonoff, Fréchet, Leray, Borsuk, Steinhaus, Mazurkiewicz, Kuratowski, Knaster, Krasnoselskij, Ky Fan, Klee, Caristi, Kirk, Browder, Dugundji, Granas, and many others.

J. Schauder himself set down this problem in 1927 and 1930 respectively and had it published in: Math. Zaitschrift and Studia Mathematica. The problem gained the importance when it was put forward by StefanBanach in 1930 at the World congress of mathematicians in Moscow.

First positive answer for locally convex space was given by Tychon off in 1935. It was J. Schauder who presented (personally) Tychonoff's paper in Zbl. für Math. 12 (1936), with number $\mathbf{3 0 8}$.
} 
Proposition 2.2 (Extension and solution of Schauder's problem). Let D be a nonempty convex subset in linear topological space $X$ and suppose that $T: D \rightarrow D$ is a continuous mapping. Then $T$ has at least countable or finite fixed points in $D$.

Proof. (Application of Theorem 2.2). From Proposition 2.1 (for convex lower semicontinuous function $B:=D \rightarrow \mathbb{R}_{+}^{0}$ ) inequality (B) holds on nonempty compact convex set $D$, thus direct it follows that $B$ satisfies the condition of lower BCS-convergence, also, i.e., $D$ and $B$ satisfy all the required hypothesis in Theorem 2.2. Applying Theorem 2.2, in this case, we obtain that $T$ has at least countable or finite forked points $\xi_{k} \in D$, such that $B\left(T\left(\xi_{k}\right)\right)=B\left(\xi_{k}\right)$. This implies, form the facts of $B, T\left(\xi_{k}\right)=\xi_{k}$. The proof is complete.

We are now in a position to formulate our following known applications. In this sense we obtain three fundamental famous principles of Brouwer, Banach and Schauder.

Theorem 2.5 (General Brouwer Theorem). Suppose that $C$ is a nonemty convex, compact subset of $\mathbb{R}^{n}$, and that $T: C \rightarrow C$ is a continuous mapping. Then $T$ has at least countable or finite fixed points in $C$.

In this sense, as a direct consequence of Theorem 2.5, we obtain the following well-known Brouwer's theorem.

Theorem 2.6 (Brouwer, [1912]). Suppose that $C$ is a nonempty convex, compact subset of $\mathbb{R}^{n}$, and that $T: C \rightarrow C$ is a continuous mapping. Then $T$ has a fixed point in $C$.

We also have, as an immediate and direct consequence of Theorem 2.2, as a version of the Schauder fixed point theorem.

Theorem 2.7 (Schauder, [1930]). Let $C$ be a nonempty, compact, convex subset of a Banach space $X$, and suppose $T: C \rightarrow C$ is a continuous operator. Then $T$ has a fixed point in $C$.

We notice that this statement is a direct translation of the Brouwer fixed point theorem to Banach spaces.

Proof. Since $C$ is a convex and compact subset of Banach space, from Theorem 2.1 (Ordering Principle) and Theorem 2.2, we obtain this statement.

Theorem 2.8 (General Schauder Theorem). Let $C$ be a nonempty, compact, convex subset of a Banach space $X$, and suppose $T: C \rightarrow C$ is a continuous operator. Then $T$ has at least countable or finite fixed points in $C$.

This statement is a direct consequence of Theorem 2.1 (Ordering Principle) and Theorem 2.2. Also, the following result is a direct consequence of Theorem 2.1 (Ordering Principle) and Theorem 2.8. 
Answer to Schauder's problem is affirmative. From the preceding statements and some further facts we are now in the position to formulate the following solution which is, also, an extension of Schauder problem.

Theorem 2.9 (Answer is yes for Schauder's problem). Let $C$ be a nonempty convex compact subset of a linear topological space $X$ and suppose that $T$ : $C \rightarrow C$ is a continuous mapping. Then $T$ has a fixed point in $C$.

This result is a special case of the former Proposition 2.2. Thus the proof of this statement we omit. For the first proof of Theorem 2.8 see Tasković [1998].

Annotation. Let us emphasize that the above solution for the Schauder's problem was presented by Tasković in 1998. Another different solution for the same problem was published by R. Cauty in 2001, three years later after M. R. Tasković. See: Tasković [1998] and Cauty [2001]! Also see: Rus [1999].

Generalized Peano's Theorem. Further we give an application of General Schauder fixed point theorem to differential equations. As a parallel and contrast to the Picard-Lindelöf theorem we consider the initial value problem of the form as

$$
x^{\prime}(t)=f(t, x(t)), \quad x\left(t_{0}\right)=y_{0},
$$

on $\left[t_{0}-c, t_{0}+c\right]$. Geometrically, (5) means that we are looking for a curve which satisfies the differential equation and passes through $\left(t_{0}, y_{0}\right)$. At the end points $t=t_{0} \pm c$, where $x^{\prime}(t)$ is to be interpreted as the appropriate one-sided derivative.

Proposition 2.3. (Peano [1890], Tasković [2012]). Let there be given real numbers $t_{0}$ and $y_{0}$, and the rectangle of the form as

$$
Q_{b}:=\left\{(t, x) \in \mathbb{R}^{2}:\left|t-t_{0}\right| \leq a,\left|x-y_{0}\right| \leq b\right\}
$$

where $a$ and $b$ are fixed positive numbers. Suppose that $f: Q_{b} \rightarrow \mathbb{R}$ is continuous and bounded with the following condition of the form as

$$
|f(t, x)| \leq K \quad \text { for all } \quad(t, x) \in Q_{b},
$$

and fixed $K>0$. Set $c:=\min \{a, K / b\}$. Then the initial value problem (5) has at least countable or finite continuously differentiable solutions on $\left[t_{0}-c, t_{0}+c\right]$.

Proof. (Application of Theorem 2.8). In addition to initial value problem (5), we also consider the integral equation of the form as

$$
x(t)=y_{0}+\int_{t_{0}}^{t} f(s, x(s)) d s,
$$

and next write this as the operator equation $x=T(x)$, for $x \in M \subset X$, where $X:=C\left(\left[t_{0}-\right.\right.$ $\left.\left.c, t_{0}+c\right]\right), M=\left\{x \in X:\left\|x-y_{0}\right\| \leq b, \quad\right.$ and $\left.\quad\|x\|=\max _{t_{0}-c \leq t \leq t_{0}+c}|x(t)|\right\}$. The set $M$ is closed, convex, and bounded in $X$; also from this it follows $T(M) \subset M$. Since the operator $T$ is compact,we have that Tasković fixed point theorem (Theorem 2.8) implies the existence at least countable or finite solutions $x=T(x), x \in M$. The proof is complete.

As an immediate consequence of Theorem 2.3 we obtain the following direct generalization of Caristi's fixed point theorem.

Theorem 2.10. Let $T$ be a self-map on a complete metric space $(X, \rho)$. Suppose that there exists a lower semicontinuous function $G: X \rightarrow \mathbb{R}_{+}^{0}$ and an arbitrary fixed integer $k \geqslant 0$ such that

$$
\rho[x, T x] \leqslant G(x)-G(T x)+\cdots+G\left(T^{2 k} x\right)-G\left(T^{2 k+1} x\right)
$$

and $G\left(T^{2 i+1} x\right) \leq G\left(T^{2 i} x\right)$ for $i=0,1, \ldots, k$ and for every $x \in X$. Then $T$ has at least countable or finite fixed points $\xi_{t}$ in $X$. 
Proof. (Application of Theorem 2.3). Let $x$ be an arbitrary point in $X$. We can show then that the sequence of iterates $\left\{T^{n} x\right\}_{n \in \mathbb{N}}$ is a Cauchy sequence. Let $n$ and $m(n<m)$ be any positive integers. From (Tk) we have

$$
\rho\left[T^{n} x, T^{m} x\right] \leqslant \sum_{i=n}^{m-1} \rho\left[T^{i} x, T^{i+1} x\right] \rightarrow 0 \quad(m, n \rightarrow \infty) .
$$

Hence, $\left\{T^{n} x\right\}_{n \in \mathbb{N}}$ is a Cauchy sequence in $X$ and, by completeness, there is $\xi_{t} \in X$ such that $T^{n} x \rightarrow \xi_{t}(n \rightarrow \infty)$. Therefore, $X$ satisfies the condition of orbital lower BCS-convergence for $B(x)=G(x)$.

We also have $B(T x) \leq B(x)$ for every $x \in X$, i.e., (B) in Theorem 2.3. Applying Theorem 2.3 we obtain a form of (Mk), i.e., $B\left(T^{2 k+1} \xi_{t}\right)=B\left(T^{2 k} \xi_{t}\right)=\cdots=B\left(T \xi_{t}\right)=B\left(\xi_{t}\right)=\alpha_{t}$ for some $\xi_{t} \in X$. Thus from (Tk) we have

$$
\rho\left[\xi_{t}, T \xi_{t}\right] \leqslant G\left(\xi_{t}\right)-G\left(T \xi_{t}\right)+\cdots+G\left(T^{2 k} \xi_{t}\right)-G\left(T^{2 k+1} \xi_{t}\right)=(2 k+1)\left(\alpha_{t}-\alpha_{t}\right)=0,
$$

i.e., $\xi_{t}=T \xi_{t}$ for some $\xi_{t} \in X$. The proof is complete.

Remark. We notice that a brief proof of Theorem 2.10 based on the Ordered Principle may be found in Tasković [1998]. We can also give a proof for special case of this statement based on Zorn's lemma as well as on Brézis-Browder ordering principle.

Also, as an immediate consequence of the preceding results we obtain the following statement of fixed point.

Theorem 2.11. Let $T$ be a self-map on a complete metric space $(X, \rho)$. Suppose that there exists a lower semicontinuous function $G: X \rightarrow \mathbb{R}_{+}^{0}$ such that

$$
\rho[x, T x] \leqslant \sum_{i=0}^{+\infty}\left(G\left(T^{2 i} x\right)-G\left(T^{2 i+1} x\right)\right)
$$

and $G\left(T^{2 i+1} x\right) \leq G\left(T^{2 i} x\right)$ for $i \in \mathbb{N} \cup\{0\}$ and for every $x \in X$. Then $T$ has at least countable or finite fixed points $\xi_{t}$ in $X$.

Proof of Theorem 1.1. (Application of Theorem 2.2). Let $x$ be an arbitrary point in $X$. The function $B: X \rightarrow \mathbb{R}_{+}^{0}$ is lower semicontinuous and satisfies, by $(\varphi), B(T x) \leq B(x)$ for every $x \in X$, i.e., the inequality (B) in former Theorem 2.2 .

Applying results to the sequence $\left\{B\left(T^{n} x\right)\right\}_{n \in \mathbb{N}}$ we obtain that $B\left(T^{n} x\right) \rightarrow 0(n \rightarrow \infty)$, and thus $X$ satisfies the condition of orbital lower BCS-convergence with the inequality (B). Applying Theorem 2.2 we have $B\left(T \xi_{t}\right)=B\left(\xi_{t}\right):=\alpha_{t}$ for some $\xi_{t} \in X$. Also, from $(\varphi)$, for $\alpha_{t} \neq 0$ we have

$$
\alpha_{t}=B\left(\xi_{t}\right)=B\left(T \xi_{t}\right) \leqslant \varphi\left(B\left(\xi_{t}\right)\right)<B\left(\xi_{t}\right)=\alpha_{t}
$$

for some $\xi_{t} \in X$. This is a contradiction, which means that $\alpha_{t}=0$, i.e., $\xi_{t}=T \xi_{t}$ for some $\xi_{t} \in X$. The proof is complete.

Remarks. An important event. We notice that the preceding facts and consequences are an affirmation that Monotone Principle of Forked Points is a natural extension of the Monotone Principle of Fixed Point, and Localization Monotone Principle of Fixed Point.

Recently, 15 years later appeared Localization Monotone Principle in 1985, in connection with this Suzuki [J. Math. Anal. Appl. 253 (2001), 440-458, Theorem 1, p. 451] which proved a very special case of Localization Monotone Principle of Fixed Point.

\section{REFERENCES}

1. E. Zermelo, Beweis dass jede Menge wohlgeordnet werden kann, (Aus einem an Herrn Hilbert gerichteten Briefe), Math. Ann., 59 (1904), 514-516.

2. E. Zermelo, Neuer Beweis für die Möglichkeit einer Wohlordnung, Math. Ann., 65 (1908), 107-128.

3. M. Zorn, A remark on method in transfinite algebra, Bull. Amer. Math. Soc., 41 (1935), 667-670.

4. M. Zorn, Idempotency of infinite cardinals, Univ. Calif. Publ. in Math., Seminar Reports (Los Angeles), 2 (1944), 9-12. 
5. M. R. Tasković, Theory of transversal point, spaces and forks, Fundamental Elements and Applications, Monographs of a new mathematical theory, VIZ-Beograd 2005, (In Serbian), 1054 pages. English summary: 1001-1022.

6. M. R. Tasković, The Axiom of Infinite Choice, Math. Moravica, 16-1 (2012), 77-94.

Remark. The complete list of references of this paper is from the paper by M.R.Tasković: Transversal theory of fixed point, fixed apices and forked points, Math. Moravica, 14-2 (2010), 19-97.

For the Fork Theory, see: https://payhip.com/milanrtaskovic

Milan R. Tasković

FACUlty of Mathematics

P.O. Box 550

11000 BEOGRAD

SERBIA

Home address:

Milan R. TASKović

NeHruova 236,

11070 BELGRADE

SERBIA

E-mail address: andreja@predrag.us 